\title{
Interprofessionalität - ein Thema für Hausärzte?
}

\author{
Peter Strohmeier, Philipp Zinsser, Peter E. Schlageter
}

Dres. med., Fachärzte für Allgemeine Innere Medizin

Interprofessionalität - ein Schlagwort. Bei der Bearbeitung des Themas in unserem hausärztlichen Qualitätszirkel Oberwil (QZO, gegründet 1996) und nach ausführlicher Diskussion unter den Mitgliedern haben wir den Eindruck gewonnen, dass die «Interprofessionalität» von vielen «bearbeitet» wird, dass aber von ärztlicher Seite zu diesem Thema bisher erstaunlich wenig zu hören ist. Wir stellen im Folgenden unsere Überlegungen dar.

\section{Weshalb sollen wir uns zur Inter- professionalität äussern?}

Wir Ärzte müssen unsere Positionen einbringen Viele Akteure im Gesundheitsbereich befassen sich mit dem Thema Interprofessionalität. Es werden Vorstellungen über die eigene Rolle bei der Betreuung von Patienten entwickelt. Man preist die eigenen Leistungen an und versucht damit, neben dem Behandlungsziel die eigene Daseinsberechtigung festzuhalten. Häufig geht es darum, Leistungen, die bisher nur auf ärztliche Verordnung von den Krankenkassen bezahlt wurden, in Zukunft in eigener Regie ohne Verordnung ausführen und abrechnen zu können. Ob dabei immer der Patientennutzen im Vordergrund steht, scheint uns fraglich.

\section{L'interprofessionnalité}

L'interprofessionnalité - un mot-clé. Lors du traitement de ce thème dans le cadre de notre cercle de qualité des médecins de famille Oberwil (QZO, constitué en 1996), et après une discussion approfondie entre les membres, nous avons acquis l'impression, que "l'interprofessionnalité» est "traitée» dans bien de milieux, mais qu'on entend étonnamment peu sur ce thème de la part des médecins. De façon générale, la collaboration interprofessionnelle est particulièrement importante quand il s'agit de patients polymorbides. Le médecin de famille, à juste titre, est souvent considéré comme un spécialiste de la complexité. Les différentes formes de collaboration avec les médecins spécialistes et autres professionnels de la santé (infirmiers/ères de soins à domicile, physiothérapeutes, psychologues, nutritionnistes, cliniques, etc.) font part de son travail quotidien. Pour garantir des soins de santé de grande qualité, il faut un ordonnateur et coordinateur des prestations à effectuer. Dans notre article, nous présentons nos considérations sur le rôle du médecin de famille, qui nous paraît central et essentiel.
Im Gegensatz zu den übrigen Berufsgruppen ist von ärztlicher Seite deutlich weniger zum Thema Interprofessionalität zu hören. Wird die Interprofessionalität ohne unser Zutun definiert und ausgehandelt? Riskieren wir, am Schluss, wenn andere die «Spielregeln» definiert haben, bei diesem wichtigen Thema nur noch Akzentverschiebungen bewirken zu können?

Beim Bearbeiten dieses Themas haben wir festgestellt, dass dieser, unser erster Eindruck nicht zutrifft. Sowohl FMH [1] als auch Haus- und Kinderärzte Schweiz (MFE) befassen sich mit dem Thema. Speziell MFE bearbeitet das Thema intensiv. Ob mit dem erwünschten Erfolg, können wir nicht abschätzen. Das Beispiel der geplanten Kampagne zur Darmkrebsprophylaxe bestätigt leider etwas unsere Befürchtung, dass andere den «Takt angeben» und wir gezwungen werden, so gut wie möglich «mitzuspielen» [2]. Schon im Vorfeld der ange-

\section{Wird die Interprofessionalität ohne unser Zutun definiert und ausgehandelt?}

strebten interprofessionellen Zusammenarbeit ist die Kommunikation zwischen den verschiedenen Berufsgruppen spärlich bis fehlend. Ohne Kommunikation werden die Patienten aber nicht «Nutzer», sondern «Opfer» jeder Form von Interprofessionalität werden.

\section{Was wollen wir erreichen?}

\section{Der Lead gehört in ärztliche Hände}

Nach unserer Erfahrung handelt es sich bei denjenigen Patienten, bei denen die interprofessionelle Zusammenarbeit von besonderer Bedeutung ist, um polymorbide Patienten, wie sie für die Hausarztpraxis typisch sind. Nicht zu Unrecht wird der Hausarzt als Spezialist für Komplexität betrachtet. Auch wenn sich in Zukunft mehrere "Leistungserbringer» eigenverantwortlich um diese Patienten kümmern sollen, braucht es jemanden, der dieses interprofessionelle Team leitet und koordiniert.

Dank der breiten und profunden Ausbildung halten wir den Arzt für diese Aufgabe für prädestiniert [3]. Dabei geht es nicht um die Verteidigung einer Machtposition, 


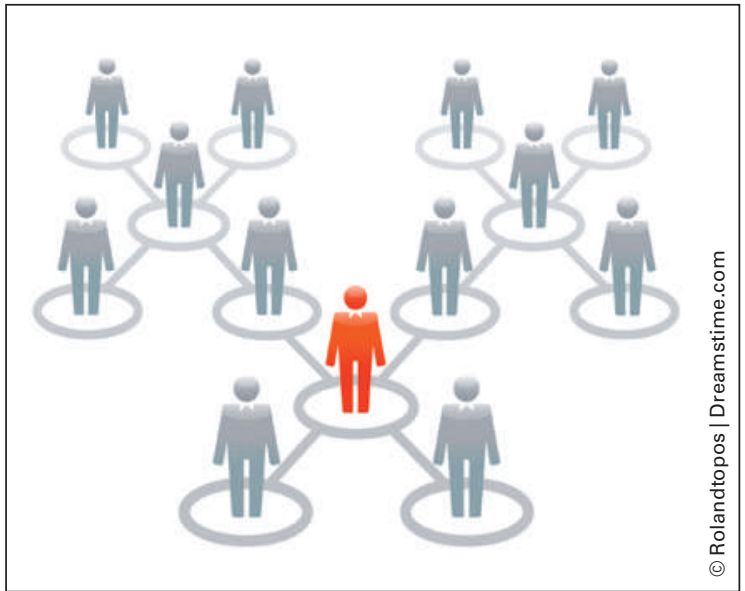

Auch wenn sich mehrere "Leistungserbringer» um Patienten kümmern, braucht es jemanden, der dieses interprofessionelle Team leitet und koordiniert.

sondern um Anerkennung einer allgemein bekannten Tatsache: Ohne entsprechende Koordination und Führungsverantwortung ist bei vielen gleichberechtigten Akteuren das Chaos vorprogrammiert. Hierin unterscheidet sich die Medizin nicht von anderen Bereichen aus der Geschäftswelt: Kein Haus wird ohne Bauführer gebaut.

1 Schlup J. Ja zum Ausbau der Interprofessionalität Nein zur Initiative. Schweiz Ärztezeitung; 2014;95(8):277.

Zirbs Savigny B. Interprofessionalität will gelernt sein. Primay Care. 2015;15(17):293.

3 Schlup J. Klare Kompetenzregelungen sind für uns unabdingbar. Synapse. 2015;(2):6.

4 Quinto C. Interprofessionalität-Notmassnahme oder Königsweg? Synapse. 2015; (2):1-3.

5 Mayo G. Über die Abwertung der eigentlichen ärztlichen Leistung in einer industrialisierten Medizin. Synapse 2015;(4):1-3.

6 Schlageter PE, Strohmeier P. Wir stehen ein für eine effiziente und respektvolle Zusammenarbeit mit anderen Gesundheits berufen. Synapse. 2015;(2):13.

Korrespondenz: Dr. med. Peter Strohmeier Vorderbergstrasse 83

CH-4104 Oberwil

pe.strohmeier[at]bluewin.ch über die Interprofessionalität die wichtigsten Mitarbeiter, die medizinischen Praxisassistentinnen zu wenig wahrgenommen werden. Einige Leistungen, die heute von den «Interprofessionalisten» eingefordert werden, können durch die MPA nach entsprechender Ausbildung abgedeckt werden. Neben der Fachkompetenz ist die kontinuierliche Zusammenarbeit zwischen Arzt und MPA von unschätzbarem Nutzen.

\section{Was erwarten wir?}

\section{Engagierte Standespolitiker und (Haus-)Ärzte}

Ganz allgemein wünschten wir uns von der FMH und von MFE ein proaktives Vorgehen. Insbesondere aber auch vermehrten Positionsbezug in der Öffentlichkeit mit Betonung der zentralen Rolle der Hausärztinnen/ -ärzte bei der Interprofessionalität. Neben dem Engagement der Standespolitiker fordern wir auch das Engagement jedes einzelnen Hausarztes. Wenn wir den Lead bei der Interprofessionalität verlangen, heisst dies auch, dass wir dieser Aufgabe gerecht werden wollen. Denn die breite und profunde Ausbildung ist nur nützlich, wenn der Leader erreichbar ist.

\section{Was befürchten wir?}

\section{Weniger Zeit für die Patienten - der Hausarzt wird zum Manager abgewertet}

Für uns Hausärzte ist das Wort «Interprofessionalität» ein Schlagwort für eine Arbeitsweise, die wir schon lange kennen. Schon heute arbeiten wir mit vielen Fachleuten zusammen. Sei es mit ärztlichen Spezialisten, sei es mit anderen medizinischen Berufsleuten (Kliniken, Pflegeinstitutionen, Spitex, Physiotherapeuten, Psychologen, Ernährungsberaterinnen usw.).

Als Hausärzte befürchten wir, durch den notwendigen Koordinationsaufwand Zeit und Energie für die Patientenbetreuung zu verlieren $[4,6]$. Damit wird die ärztliche Tätigkeit abgewertet und die Verwaltung aufgebläht. Ein Muster, das wir bereits jetzt in vielen Bereichen des Gesundheitswesens sehen [4-6].

Die persönliche Betreuung der Patienten muss die Hauptaufgabe des Hausarztes bleiben!

\section{Illusionen?}

\section{Botschaft an die Gesundheitspolitiker}

Es droht der Mangel an Hausärzten. Dieses Problem ist nicht mit Interprofessionalität zu lösen. Wenn wir in der Schweiz auch in Zukunft eine hochstehende medi-
Für uns Hausärzte ist das Wort «Interprofessionalität» ein Schlagwort für eine Arbeitsweise, die wir schon lange kennen.

zinische Versorgung wollen, müssen wir vor allem und Gesundheitsfachkräften investieren. Es zeichnet sich auf allen Ebenen ein Mangel an Fachleuten ab.

Ganz falsch ist die Vorstellung, dank Interprofessionalität die Gesundheitskosten reduzieren zu können. Schliesslich werden nicht weniger Leistungen erbracht, die Leistungen werden lediglich umverteilt. Es entsteht ein erheblicher administrativer Mehraufwand zur Steuerung der Abläufe.

Insgesamt befürchten wir einen Qualitätsverlust im schweizerischen Gesundheitswesen.

\section{Danksagung}

Unser besonderer Dank gilt unseren Kolleginnen und Kollegen im Qualitätszirkel Oberwil, Dres. med. J. Babics, L. Kober, M. Loeliger, A. Lyrer, O. Mohr, W. Nef, E. Ramseier und G.-C. Serena, für ihre Beiträge zum vorliegenden Artikel. dringend in die Ausbildung von Ärzten und weiteren 